\title{
BabyTigers-99: Osaka Legged Robot Team
}

\author{
Noriaki Mitsunaga and Minoru Asada \\ Dept. of Adaptive Machine Systems, Osaka University, Suita, Osaka, 565-0871, Japan
}

\section{Introduction}

Our interests are learning issues such as action selection, emergence of walking, and self localization without 3D-reconstruction. We implemented teaching, self localization without 3D-reconstruction and embodied trot walking.

Our embodied walking showed the fastest movement in the all nine teams. We got second place in the RoboCup Challenge. Although due to the fatal bug revealed in the game, we lost in the semi-final game and we got the fourth place in the competition.

\section{Team Development}

Team Leader: Minoru Asada

Team Members:

Minoru Asada

- Osaka University

- Japan

- Professor

- Attended the competition

Noriaki Mistunaga

- Osaka University

- Japan

- Ph.D candidate

- Attended the competition

Tatsuro Nohara

- Osaka University

- Japan

- Master course student

- Attended the competition

Web page http://www.er.ams.eng.osaka-u.ac.jp/

\section{Complete Robot Architecture}

Since everything changes rapidly in the real world, we consider it important for the robot to make fast decisions. Then we adopted to execute the learned movements rather than do planning during the games.

We wrote a program which consists of three objects. One is for the vision, one for the walking and head movements, and one for cognition and decision making. 


\section{Vision}

In the RoboCup Legged Robot League field [2], seven colors (aqua-blue, yellow, orange, blue, red, pink, green) are used and robots need to detect and discriminate them. The Sony's legged robot has the color detection facility in hardware that can handle up to eight colors at frame rate. To detect colors with this facility, we need to specify each color in terms of subspace in $Y U V$ color space. $Y U V$ subspace is expressed in a table called Color Detection Table(CDT). In this table, $Y$ are equally separated into 32 levels and in each $Y$ level we specify one rectangle $\left(u_{\min i}, v_{\min i}\right),\left(u_{\max i}, v_{\max i}\right)(i=1, \ldots, 32)$.

In order to make CDTs, we used the same method used in last year. Briefly, 1) take an image which includes the object to detect with the robot, 2) specify pixels to detect with GUI program and make a list of YUV to detect, 3) order the program to classify each pixel according to the $\mathrm{Y}$ level as they are classified in CDT and make a bounding box of UV in each level, 4) check if detection satisfies the need and if not do 1) again. Iterate these procedure for each color with several images.

If some parts of an object are detected by a CDT, but still other parts of it remain not detected, one can extend the CDT with continuousness of color in YUV space. We also made this kind of CDT expansion tool and used it (Fig.1).

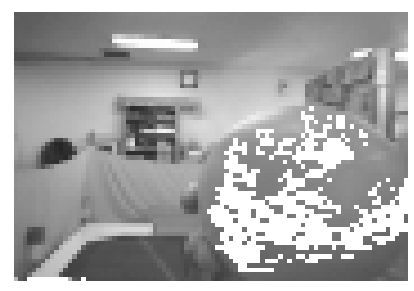

(a) A partially detected image by CDT

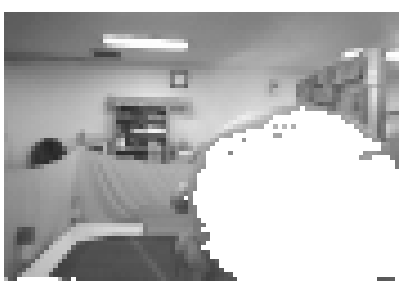

(b) Mostly detected image by expanded CDT

Fig. 1. An example of expanding CDT

Objects recognition was done by extraction of connected areas with 8-neighbor method in the color detected images. After connected areas are extracted, object recognition, including landmarks (all landmarks are consisted of two colors) recognition are done by concatenating the areas. To overcome noises in the image, the order was determined empirically. Details of 1)-4) and objects recognition are described in [1]. 


\section{Control}

Since it is important to move fast and efficiently, we developed an embodied trot walking, which enables simple controlling program and fast movement. Details will appear elsewhere.

For the posture control we used getting up program designed by SONY. We wrote down the program to sense of falling down, and called the SONY's recovering program when needed.

Head movement for searching and tracking the ball and observing landmarks about 180 degrees have been implemented beforehand. Also we prepared six discrete movements, forward, left forward, right forward, left turn, right turn, and chase the ball for 2.4 seconds (four walking periods). Since we decided to use teaching for behavior acquisition, we did not implement behavior such as avoiding obstacles or keeping its own goal.

\section{Localization}

In order to determine the behavior to take, it is important to localize itself in the soccer field. Location estimation with occupancy grids is time and memory consuming and it is difficult to do dead recogning with trot walking due to slippage. Although the 180 degree view is unique in most locations of the RoboCup Legged Robot League field, the robot has to pan or memorize how the landmarks were seen for 3-D reconstruction, due to the limited view angle of the camera.

To overcome such difficulties, we used the direction of the landmarks from the robot for its localization. The robot sees landmarks panning the camera and calculates in which direction they are and assigns the quantized direction to each landmark. Depending on the directions of the landmarks and the ball, the robot behaves as the human trainer taught. To reduce the time for panning, we also implemented estimation of landmarks. The robot only observes if estimation and current view of landmarks are not sufficient to determine an action to take.

\section{$7 \quad$ Strategy}

We used two kinds of programs. One is to demonstrate the fast trot walking, which only chases the ball. The other is to play back the taught behavior. Therefore our strategy mostly depended on the human trainer. Basic teaching policy was, 1)if the ball can be seen, 1-a) chase the ball if the target goal can be seen, 1-b) otherwise, turn around the ball as to see the target goal, 2)if the ball can not be seen, 2-a) if near the own goal, turn outside not to make own goal, 2-b) otherwise, turn to center of the field expecting to see the ball. Unfortunately, we have not considered team works in the teaching yet. 


\section{Special Team Features}

We have proposed and implemented a new method for decision making based on information criterion. The basic idea is to construct a decision tree and prediction trees of the landmarks, which enable a robot with a limited visual angle to localize itself in the environment with fewer observations. The number of pannings (observation) are reduced by predicting the locations of landmarks and judging if the current observation and prediction of landmarks are sufficient for the decision making. We used a teaching method to collect example data for making the decision and prediction trees. Details will appear elsewhere.

\section{Conclusion}

We implemented an embodied trot walking and showed fast movements but had difficulty in making small or steady movements. Embodied steady static walking which can easily combined with dynamic walking should be developed.

We employed the direct teaching method for learning. For the self localization, we used quantized direction of landmarks from the camera rather than 3 -D reconstruction. We also implemented a decision making method to see or to take an action dependent on estimation. Team work and self learning are future issues.

\section{References}

1. N. Mitsunaga, M. Asada, and C. Mishima. Babytigers-98: Osaka legged robot team. In M. Asada and H. Kitano, editors, RoboCup-98: Robot Soccer World Cup II, pages 498-506. Springer, Lecture Note in Artificail Intelligence (1604), 1999.

2. M. Veloso, W. Uther, M. Fujita, M. Asada, and H. Kitano. Playing soccer with legged robot. In Proceedings of the 1997 IEEE/RSJ International Conference on Intelligent Robots and Systems, volume 1, pages 437-442, 1998. 